\title{
A Survey on Data Integrity Methods in Cloud Storage
}

\author{
G. Anil Kumar and A. S. Poornima
}

\begin{abstract}
Cloud computing offers different kind of services to the end user, so that end user can store the data and access it on demand where ever they need it. Now days IT industries are outsourcing their data by storing it remotely in cloud for reducing the load on local storage there by reducing the hardware, software and maintenance cost. In spite of these benefits, the major problem with data storage is that there is no guarantee of data consistency and integrity, which has become a major hurdle for using the services offered by cloud. So this paper surveys existing schemes that proves data integrity and retrievability.
\end{abstract}

Keywords-POR, PDP, Data integrity, Message authentication code.

\section{INTRODUCTION}

Data integrity generally means assuring the data accuracy and consistency of data during its life time that is ensuring that the current data is same as original data when it was recorded [1]. When end user stores the data in the cloud, he has to check periodically for integrity of the data which is called as Remote Integrity Check (RIC). In local data integrity check for checking adversarial errors collision resistance hash function and message authentication code is used. Remote data integrity check can be done by checking the stored data files periodically, but the problem with this is the data file is not available at the time of verification. Two early approaches of proof of storage are RSA based and MAC based methods, RSA scheme is efficient in storage and communication, both schemes suffers from storage overhead. Many of the cryptographic concepts are associated with remote integrity check such as Proof Of Retrievability (POR), Proof of Data Possession (PDP) [1][2]. This paper presents the survey of these two schemes and several methods used in the schemes. Rest of the paper is organized as follows, Section 2 methods in Proof of Retrievability, Section 3 methods in Proof data possession, followed by conclusion in section $4 .^{1}$

\section{PROOF OF RETREIVABILITY(POR)}

Earlier Non homomorphic Message Authentication Code(MAC) schemes suffers from communication cost, it was then improved to overcome this drawback by Ateniese et al, et al proposed linearly homomorphic MAC, which consists of

Published on November 10, 2016

G. A. Kumar is with Siddaganga Institute of Technology, Tumkur, Karnataka, India (e-mail: aaniltumkur@gmail.com).

A. S. Poornima was Siddaganga Institute of Technology, Tumkur, Karnataka, India (e-mail: poornimaarvind9@gmail.com) four algorithms for generating (keygen), sign, combine and verify. A POR scheme named as POS1 was constructed as shown below, key generating algorithm generates a public and private key pair, sign algorithm generates a tag for each block, and sends encoded file and id to cloud server, keeps id and blocks in local storage, then message and a MAC value is generated using homomorphic property, combine algorithm is used for combining the private key, id, and encoded file sends this to verifier. The verifier using private key, message, id verifies this to accept or reject.

\section{Advantage: Efficient in computation and communication.} This scheme is secure and sound.

Disadvantage: Authentication tag and data blocks are of same size so storage overhead is more.

To overcome this drawback, a scheme named linearly predicate homomorphic message authentication code was introduced. This scheme includes six algorithms such as key generation, Sign, Combine, P-sign, Verify, P-Verify. The key generation algorithm takes security parameter as input and generates public and private key pairs. The sign algorithm takes three inputs message, index and private key, gives signature as the output. This scheme should hold two conditions, linear homomorphism and predicate homomorphism Any Message Authentication Code (MAC) scheme is said to be linearly homomorphic iff the linear combination of messages can be generated without private key from message's MAC value. Any MAC scheme is said to be predicate homomorphic iff MAC value of this message can be generated without using private key from the MAC value of another message [2]. The combine algorithm holds linear homo morphic property it takes public key and a sequence of tuples which consists of index, MAC value of message, weight of the message. Message -MAC pair is the output of this algorithm. P-Sign algorithm holds predicate homomorphism property and it takes value r, public key, and message MAC as input. Produces message - MAC pair as the output. Verify is a deterministic algorithm which takes three inputs that is sequence of index and weight pairs, messageMAC pair and a private key. Output is either accepted or rejected. P-Verify is a deterministic predicate verify algorithm takes same inputs as verify algorithm along with value $r$ as the input and outputs accepted or rejected.

Advantages: Very efficient in communication and storage, practical in computation, security is proved under DiffieHellmann and secure pseudo random function assumption. 


\section{Disadvantages: For Static data only.}

\section{Provable Data Possession(PDP)}

PDP is a protocol which enables clients to the data file stored on the cloud storage is available in its original form or not [3]. The generic PDP design as shown in figure 1.1 consists of four phases, First phase is preprocessing, in this phase client builds the file's meta-data, which is kept secret and an encrypted version of data file or water marked file is sent to the cloud storage. Next is the challenge phase here client sends randomized challenge to the server to verify the data possession. In proof phase as a response to the challenge server computes proof based on request received. In verifying phase client checks the proof against meta-data stored in it. Hash function is used design a simple PDP scheme, where client generates the fixed number of random challenges, computes the related responses, and then compares with the locally stored meta-data if it matches then the cloud server has correct data file.

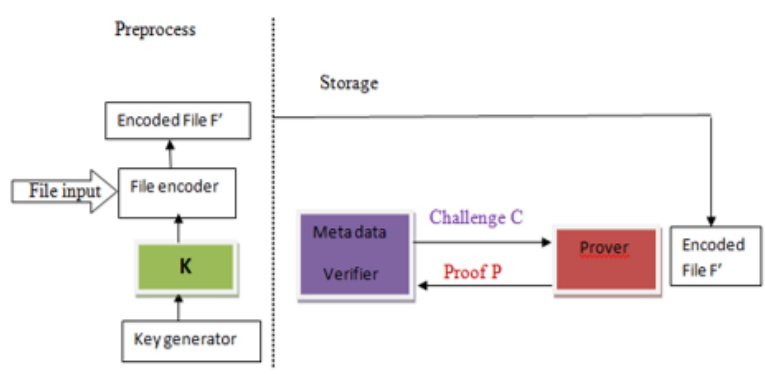

Fig. 1. Generic phases of PDP.

\section{A. A Zero Knowledge Scheme For PDP In Cloud Storage}

Zero knowledge PDP is a special kind of interactive proof mechanisms [4]. The main idea of this scheme is that prover needs to convince the verifier that the statement is true without revealing any of his secrets, the verifier and the prover exchanges the interactive protocol, verifier will not learn anything from interaction with prover [3][4].

By taking this advantage of Zero knowledge scheme a novel approach was introduced and it is based on GPS. GPS is a public key based protocol for resource constraint devices. The entities that participate are client(C), user(U),cloud service provider(CSP). After preprocessing client stores the data file in the cloud server, users shares the data with the client will send challenge to cloud server to prove the data possession. Verification in this model consists of five algorithms, to create multileveled equations, it may be necessary to treat the equation as a graphic and insert it into the text after your paper is styled.

- KeyGen algorithm takes security parameter as input and generates public and private key pairs as output, where public key is the elliptical curve point.
- SetUp algorithm accepts data file (binary file) and public key as the input and generates file identifier and public elements.

- GenChal algorithm produces the randomized challenge.

- ChalProof algorithm takes challenge and original version of the file as input, then produce proof $\mathrm{P}$ as the output.

- Verify algorithm takes proof, private key and public elements as input and produces output as either accepted or rejected.

Advantages: Data leakage attacks are avoided, it supports public verifiability, storage and communication costs are constant.

Disadvantage: In case of large volume of data, PDP verification of data will be slower.

\section{B. Cooperative PDP Scheme}

- Cooperative PDP Scheme consists of two algorithms and an interactive proof system [5].

- KeyGen algorithm takes security parameter as the input and produces secret key or Public and secret key pair as the output.

- TagGen algorithm takes a file, secret key and cloud service providers set, then produces secret tag, set of verification parameters and index hierarchy, set of all tags as the output.

- Interactive Proof system gives a rule for interaction between cloud service provider and a verifier.

Advantages: Satisfies the zero knowledge property and also completeness property, it is collision resistant.

Disadvantages: Only for Static data files.

\section{Dynamic Provable Data Possession(DPDP)}

For most of the applications, it is necessary to consider the dynamic scenario that is, client keeps interacting with the data in the cloud storage mean while it should guarantee the data possession. To meet this requirement scalable PDP scheme [6] was introduced. But this scheme worked for pre defined number of operations within a limited set of operation. Two approaches were used in DPDP. Rank based authenticated skip list (RBASL) each node in this list has a rank which indicates the number of leaf nodes which are reachable from the particular node. This list allows to search based on the indices of blocks. Ranks will handle the problem of Block numbers which arises in PDP schemes.

Advantages: It reduces overheads as it is light weight. It allows the clients to randomly check the data integrity.

Disadvantages: Communication overhead is more.

Second approach is based on balanced update tree [7] this scheme uses MAC (Message Authentication Code to check data integrity, the balanced update tree goes on growing as updates increases, on insertion and deletion indices of the data blocks has to be recalculated. 
Advantage: In this scheme server need not maintain the Data structure to be linear in size with the data which is outsourced. Disadvantage: client keeps on updating the tree, which is an overhead.

Flexible Dynamic Provable Data Possession (Flex DPD) is another approach which uses Flex list as the data structure. It uses homomorphic verifiable tags, a single tag can be obtained from combining many tags corresponding to combined multiple blocks [8].

Advantage: Reduced time complexity that is PDP takes $\mathrm{O}(1)$, where as this scheme takes $\mathrm{O}(\operatorname{logn})$.

Disadvantage: FlexPDP is not considered for replicated and distributed servers. No erasure code is supported.

\section{Discussion on different PDP and POR schemes:}

By examining these different procedures utilized as a part of PDP and POR plans helps to know which more pertinent plan for per user's application. The underneath table gives us correlation of various varieties of PDP and POR schemes.

\begin{tabular}{|c|c|c|c|}
\hline $\begin{array}{l}\text { PDP/POR } \\
\text { Data Integrity } \\
\text { Schemes }\end{array}$ & Technique used & Advantages & Disadvantages \\
\hline POR: POS1 & $\begin{array}{l}\text { Linear Homomorphic Message } \\
\text { Authentication code }\end{array}$ & $\begin{array}{l}\text { Efficient in computation and } \\
\text { communication. } \\
\text { This scheme is secure and sound }\end{array}$ & $\begin{array}{l}\text { Authentication tag and data blocks are } \\
\text { of same size so storage overhead is } \\
\text { more. }\end{array}$ \\
\hline POR:POS2 & $\begin{array}{l}\text { Predicate message authentication } \\
\text { code }\end{array}$ & $\begin{array}{l}\text { Very efficient in communication and } \\
\text { storage, practical in computation, security } \\
\text { is proved under Diffie-Hellmann and } \\
\text { secure pseudo random function } \\
\text { assumption. }\end{array}$ & For Static data only. \\
\hline PDP & 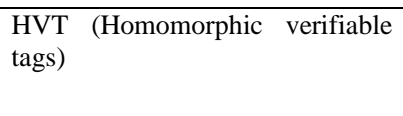 & $\begin{array}{l}\text { For security RSA is used, Public } \\
\text { verifiability is allowed }\end{array}$ & $\begin{array}{l}\text { Brute force approach is used for } \\
\text { searching blocks, For static data only } \\
\text { not for dynamic block of data. }\end{array}$ \\
\hline $\begin{array}{l}\text { Zero } \\
\text { Knowledge } \\
\text { PDP }\end{array}$ & $\begin{array}{l}\text { Interactive proof system based on } \\
\text { Girualt-Poupard } \quad \text {-Stern (GPS) } \\
\text { protocol }\end{array}$ & $\begin{array}{l}\text { Data leakage attacks are avoided, it } \\
\text { supports public verifiability, storage and } \\
\text { communication costs are constant. }\end{array}$ & $\begin{array}{l}\text { In case of large volume of data, PDP } \\
\text { verification of data will be slower. }\end{array}$ \\
\hline $\begin{array}{l}\text { Cooperative } \\
\text { PDP }\end{array}$ & Multi cloud CPDP model & $\begin{array}{l}\text { Satisfies the zero knowledge property and } \\
\text { also completeness property, it is collision } \\
\text { resistant. } \\
\text { Search complexity is reduced since it uses } \\
\text { hierarchical hash index }\end{array}$ & $\begin{array}{l}\text { Only for Static data files. } \\
\text { Complexity is increased due to } \\
\text { combiner module }\end{array}$ \\
\hline Dynamic PDP & $\begin{array}{l}\text { 1.RBASL } \\
\text { 2. Balance update tree }\end{array}$ & $\begin{array}{l}\text { 1. It reduces overheads as it is light } \\
\text { weight. It allows the clients to randomly } \\
\text { check the data integrity. } \\
\text { 2. Communication overhead is more. }\end{array}$ & $\begin{array}{l}\text { 1. In this scheme server need not } \\
\text { maintain the Data structure to be } \\
\text { linear in size with the data which is } \\
\text { outsourced. } \\
\text { 2. keeps on updating the tree, which is } \\
\text { an overhead }\end{array}$ \\
\hline $\begin{array}{l}\text { Flexible } \\
\text { Dynamic PDP }\end{array}$ & $\begin{array}{l}\text { Flex list and homomorphic } \\
\text { verifiable tags }\end{array}$ & $\begin{array}{l}\text { Reduced time complexity that is PDP } \\
\text { takes } \mathrm{O}(1) \text {, where as this scheme takes } \\
\mathrm{O}(\operatorname{logn}) \text {. }\end{array}$ & $\begin{array}{l}\text { FlexPDP is not considered for } \\
\text { replicated and distributed servers. No } \\
\text { erasure code is supported. }\end{array}$ \\
\hline
\end{tabular}




\section{CONCLUSION}

This paper introduces the diagram of information uprightness demonstrating plans, and breaks down the quality and downsides of various PDP and POR plans. This overview presumes that taking into account the document size and static or element information record plan can be secured the outsourced information in the cloud server.

\section{REFERENCES}

[1] Xinyu Fan,“On remote data integrity checking of the cloud storage”, 2013 University of Wollongong.

[2] Jia Xu, "Towards Efficient Proofs of Storage and Verifiable Outsourced Database in Cloud Computing”, 2012 National university Singapore.

[3] Nesrine Kaaniche "Cloud Data Storage Security based on Cryptographic Mechanisms”, 2014 Institue National des Telecommunication.

[4] Nesrine Kaaniche, Ethmane EI Moustaine, Maryline Laurent,“A Novel Zero-Knowledge Scheme for Proof of Data Possession in Cloud Storage Applications”,2014,PP 1-10.

[5] Ms.Ashwini Mandale, Prof.Shriniwas Gadage, "Cooperative Provable Data possession for integrity verification in multicloud", International Journal of Engineering Research and General Science Volume 3, Issue 2, Part 2, March-April, 2015 PP 550-555.

[6] C. Chris Erway, Alptekin Kupcu, Charalampos Papamanthou and Roberto Tamassia, "Dynamic Provable Data Possession”, Brown University, Providence RI April 24, 2012 PP 1-24

[7] Yihua Zhang and Marina Blanton, "Efficient Dynamic Provable Possession of Remote Data via Balanced Update Trees”, ASIA CCS'13, May 8-10, 2013, Hangzhou, China

\section{BIOGRAPHY}

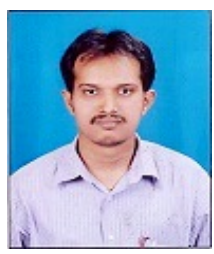

First A. Mr. G Anil Kumar is from India born in the year 1980. He perused his bachelor degree in Computer Science \& Engineering from Gulburga University, Karnataka, India and masters in Computer Science \& Engineering from Dr. MGR Educational Research Institute, Chennai, India.

$\mathrm{He}$ is working as Associate Professor in Channabasaveshwara Institute of Technology, Tumkur, India. He is having good academic and research experience in the area of Computer Networks, Unix Systems Programming, Cloud Computing with good number of publications. 